\title{
O Design Instrucional e seu uso como Arquitetura Pedagógica: Uma análise das publicações em Informática na Educação no Brasil
}

\author{
Anderson F. Barbosa ${ }^{1}$, Isabel D. Nunes ${ }^{2}$, Douglas A. T. de Menezes ${ }^{1}$, Ulrich Schiel $^{1}$ \\ ${ }^{1}$ Universidade Federal de Campina Grande (UFCG), Campina Grande - PB - Brasil \\ ${ }^{2}$ Instituto Metrópole Digital (IMD - UFRN), Natal - RN - Brasil \\ \{anderson, douglasmenezes\} @copin.ufcg.edu.br, bel@imd.ufrn.br, \\ ulrich@computacao.ufcg.edu.br
}

\begin{abstract}
Resumo. O Design Instrucional (DI) apresenta-se como uma metodologia de apoio ao processo de planejamento educacional. Tal processo, também pode ser obtido com o uso de Arquiteturas Pedagógicas (AP). Dessa forma, esse artigo tem a finalidade verificar a aplicação do DI como AP, através de um mapeamento entre as características de ambas as abordagens. Além disso, através de um mapeamento sistemático, foi caracterizado o uso do DI em Informática na Educação no Brasil. Como resultado, verificou-se a baixa quantidade de estudos relacionados à temática do DI no contexto estudado, por outro lado, o uso do DI como AP apresentou-se coerente, possibilitando, assim, a realização de novos estudos envolvendo as duas áreas observadas.
\end{abstract}

Palavras-Chave: Design Instrucional, Arquitetura Pedagógica, Informática na Educação.

\section{Introdução}

A Educação Online permite a construção do conhecimento por meio de ferramentas baseadas na web [Bittencourt e Costa 2010]. Contudo, nesse processo de construção, algumas preocupações devem ser consideradas, tais como: uso de estratégias pedagógicas, usabilidade, acessibilidade, ambientes colaborativos, acompanhamento do aprendizado e as avaliações [Hage e Aimeur 2010].

Para atender tais preocupações, os ambientes de Educação Online devem ser planejados, personalizados, implementados e melhorados a partir dos feedbacks das avaliações realizadas [Fetaji e Fetaji 2010]. Nesse processo, observa-se a existência de diferentes etapas, que podem utilizar componentes relacionados a diferentes áreas do conhecimento, como suporte ao mesmo.

Tal ideia é reforçada pelas Arquiteturas Pedagógicas (AP) através da confluência de diferentes componentes relacionados às áreas da Pedagogia e Tecnologia da Informação, de forma que, quando combinados, seja proporcionado um "suporte estruturante" para a educação [Nevado, Dalpiaz e Menezes 2007]. Seguindo o mesmo pressuposto de confluência de elementos de diferentes áreas para apoiar o processo de educacional, o Design Instrucional (DI) apresenta-se como uma metodologia que se fundamenta nas áreas das Ciências humanas, Ciências da Informação e Ciências da Administração [Filatro 2008].

Diante disso, observa-se que AP e DI são abordagens distintas, mas que podem se relacionar entre si, pois apresentam finalidades semelhantes e são compostas pela 
junção de elementos de diferentes áreas. A multidisciplinaridade das abordagens pode despertar interesse de pesquisadores, inclusive para a área da Ciência da Computação, proporcionando diversas contribuições através da aplicação de um suporte computacional em ambos os processos. Tais interesses podem ser verificados em conferências internacionais ${ }^{1}$ que apresentam o DI como um dos tópicos principais. No Brasil, os principais eventos e revista na área de Informática na Educação (IE) são organizados pela Comissão Especial em Informática na Educação (CEIE), entretanto, o termo DI não está explicitamente apresentado nas chamadas de trabalhos, diferentemente da AP que possui um workshop específico durante o Congresso Brasileiro de Informática na Educação (CBIE).

Desta forma, esta pesquisa tem a finalidade de investigar a aplicação do DI como AP, através de um mapeamento das características de ambas as abordagens. Além de caracterizar o uso do DI nas pesquisas em IE no Brasil através de um mapeamento sistemático.

\section{Pressupostos Teóricos}

Esta seção destina-se a apresentar a fundamentação teórica referente às Arquiteturas Pedagógicas (AP) e ao Design Instrucional (DI), essenciais para a compreensão do estudo realizado neste artigo.

\subsection{Arquitetura Pedagógica}

As Arquiteturas Pedagógicas podem ser definidas como estruturas capazes de pensar a aprendizagem como um trabalho artesanal, construído a partir de aspectos inerentes ao ambiente. Para isso, estas estruturas devem ser construídas a partir da confluência de diferentes componentes, tais como: abordagem pedagógica, software educacional, internet, técnicas de Inteligência Artificial, EAD, e da concepção de tempo e espaço [Carvalho, Nevado e Menezes 2005].

Segundo Nevado, Dalpiaz e Menezes (2007) a AP utiliza-se dos aspectos da vivência de experiências, de reflexões e metarreflexões do sujeito, em interação com o seu meio ambiente sócio-ecológico para construir a aprendizagem. No entanto, o papel do professor não pode ser dispensado, pois, além de ser responsável pela criação e proposição da arquitetura, ele deve prestar orientação aos alunos.

Outra definição considerada a AP como "um sistema de premissas teóricas que representa, explica e orienta a forma como se aborda o currículo e que se concretiza nas práticas pedagógicas e nas interações professor-aluno-objeto de estudo/conhecimento" [Behar 2009]. Constituindo, assim, um conjunto de elementos inter-relacionados formado pelas áreas de conhecimento: organizacional, instrucional, metodológica e tecnológica (Figura 1) [Behar, Bernardi, Silva 2009].

\subsection{Design Instrucional}

O Design Instrucional (DI) pode ser compreendido como sendo a junção dos conceitos: design e instrução. Para Filatro (2010) o termo design representa o produto de um processo ou atividade, em termos de forma e funcionalidade, com propósitos e intenções

\footnotetext{
${ }^{1}$ E-Learning 2015 e ICALT
} 
definidas; já o termo "instrução" pode ser definido como uma instrução programada ou treinamento.

Dessa forma, o Design Instrucional, que é composto conforme Figura 2, pode ser compreendido como "a ação intencional e sistemática de ensino, que envolve o planejamento, o desenvolvimento e a utilização de métodos, técnicas, atividades, materiais, eventos e produtos educacionais em situações didáticas específicas, a fim de facilitar a aprendizagem humana a partir dos princípios de aprendizagem e instrução conhecidos" [Filatro 2010].

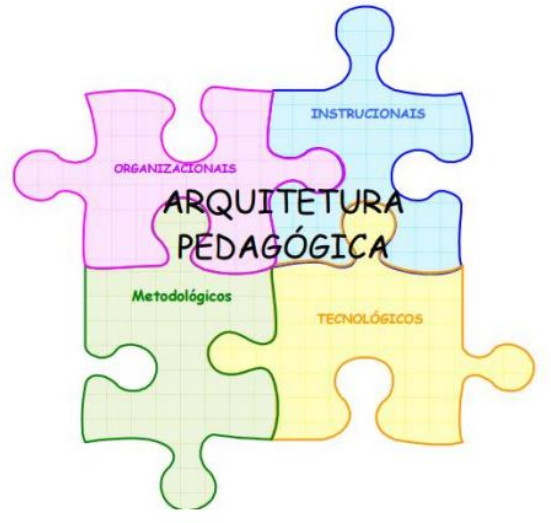

Figura 1. Elementos Inter-relacionados de uma AP

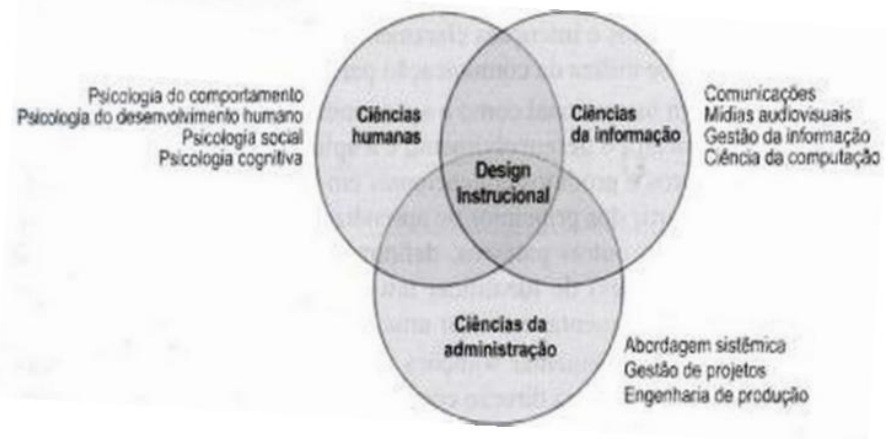

Figura 2. Fundamentos do Design Instrucional

Como reforço, Dallacosta et al. (2010) apresenta o DI como um elemento de mediação entre a educação, tecnologia e comunicação, que busca traduzir os princípios gerais da aprendizagem, do saber e da instrução em planejamento de materiais e aprendizagem.

\section{O uso Design Instrucional como Arquitetura Pedagógica}

Se observado as características do DI e da AP, verifica-se que ambas apresentam o propósito de auxiliar o processo de planejamento educacional, tendo como base a observação de aspectos inerente ao ambiente ao qual está inserido, características dos alunos, objetivos de aprendizagem, recursos de aprendizagem utilizados, dentre outros. Além disso, ambas as metodologias são compostas por elementos que norteiam as ações que deverão ser realizadas.

Segundo Behar (2009) a AP é constituída por quatro elementos, são eles: aspectos organizacionais - inclui o propósito do processo de ensino-aprendizagem, a organização do tempo e espaço, e expectativas na relação da atuação dos participantes; conteúdo - materiais instrucionais, recursos, objetos de aprendizagem, softwares, dentre outras ferramentas; aspectos metodológicos - definição das atividades, formas de apresentação, organização, interação, avaliação da sequência didática; e aspectos tecnológicos - definição do ambiente, funcionalidades, ferramentas, dentre outros.

Os elementos que constituem a AP podem ser identificados nas etapas do processo de DI através do modelo ADDIE (abreviatura em inglês para analysis, design, development, implementation e evaluation) [Filatro 2008]. Nesta pesquisa, apenas as duas primeiras etapas do modelo ADDIE terão destaque, por apresentarem finalidade semelhante as AP. Para isso, na fase de análise deve ser identificado o problema educacional e realizada uma projeção de uma solução aproximada. Enquanto, na fase do 
design deve ser realizado o planejamento e o design didático, a partir do mapeamento e sequenciamento dos conteúdos, definição de estratégias, dentre outros.

Ao observar as duas abordagens de planejamento, pode-se afirmar que as etapas de análise e design do modelo ADDIE compreendem todos os elementos da AP, conforme mapeamento realizado (Tabela 1). As etapas de desenvolvimento, implementação e avaliação do modelo ADDIE não são cobertas pelos elementos da AP.

Tabela 1. Etapas de Processos de DI x Mapeamento Elementos da AP

\begin{tabular}{|c|c|}
\hline Processo de DI (Etapas ADDIE) & Elemento da AP \\
\hline Análise & Aspectos Organizacionais \\
\hline \multirow{2}{*}{ Design } & Conteúdos \\
\cline { 2 - 2 } & Aspectos Metodológicos \\
\cline { 2 - 2 } & Aspectos tecnológicos \\
\hline
\end{tabular}

Com base nos pressupostos apresentados e mapeamento entre as abordagens verificou-se a necessidade de analisar como a temática do DI está sendo abordada pela comunidade de IE no Brasil. Tal importância foi em decorrência da identificação positiva da aplicação do DI como AP.

\section{Uso do Design Instrucional nas pesquisas em IE no Brasil}

Para identificar os estudos que abordam o uso do DI na IE no Brasil, foi utilizada a metodologia de Mapeamento Sistemático (MS), que permite investigar um determinado tema de uma maneira formal, fornecendo um subsídio real através da identificação de lacunas e resposta a questões de pesquisas [Kitchenham 2007].

A MS utilizada nesse artigo baseia-se no processo proposto por Petersen et al. (2008), composto pelas fases: definição de questões de pesquisa; realização de pesquisas por estudos primários; triagem dos documento; keywording dos resumos; e extração de dados e mapeamentos.

\subsection{Definição das questões de pesquisa}

O questionamento que motivou a realização desse mapeamento foi: "Como o DI está sendo utilizado nas pesquisas da área de IE no Brasil?”. A partir disso, foram delimitadas as seguintes questões de pesquisa:

1. Como ocorreu a distribuição das publicações que abordam o uso do DI em IE?

2. Quais as instituições que contribuíram com a realização desses estudos?

3. Qual a finalidade de uso do DI no(s) estudo(s) identificados(s)?

\subsection{Pesquisa por estudos primários relevantes}

Devido ao escopo do trabalho, optou-se por utilizar como fonte de dados as publicações organizadas pela $\mathrm{CEIE}^{2}$, a partir das publicações dos anais do Simpósio Brasileiro em Informática na Educação ${ }^{3}$ (SBIE), Workshop de Informática na Escola ${ }^{4}$ (WIE) e Revista

\footnotetext{
${ }^{2}$ Comissão Especial de Informática na Educação.

3 Simpósio Brasileiro em Informática na Educação.

4 Workshop de Informática na Escola.
} 
Brasileira de Informática na Educação ${ }^{5}$ (RBIE). Considerando a disponibilidade do acervo online, tem-se uma variação do período de publicação de acordo com a fonte (SBIE: 2001-2014; WIE: 2003-2014, exceto o ano 2004 por não estar disponível; RBIE: 1997-2014).

Para a definição dos termos da string de busca foi realizada uma busca por publicações que contivessem o termo "Design Instrucional" em seu texto. De acordo com o resultado e leituras da literatura existente, verificou-se que outros termos poderiam ser adicionados à string para que publicações que abordam a mesma temática pudessem ser selecionadas. Assim, foi criada a seguinte string de busca:

\section{("design instrucional") OR ("instructional design") $\boldsymbol{O R}$ ("learning design") OR ("projeto instrucional") OR ("desenho instrucional")}

\subsection{Triagem dos documentos}

A triagem dos documentos foi realizada através da aplicação de critérios de inclusão e exclusão aos documentos retornados pelas bases de dados.

Para este MS foram determinados os seguintes critérios de inclusão: (i) as publicações devem ser escritas em inglês ou português; (ii) as publicações devem possuir versão completa disponível na web; (iii) as publicações devem conter em seu texto o termo "Design Instrucional" ou variações utilizadas na string de busca; (iv) os estudos devem abordar o DI como enfoque principal ou secundário em destaque do estudo realizado, de forma que responda as questões de pesquisa. Enquanto os Critérios de Exclusão definidos consistem em (i) oposto dos critérios de inclusão; (ii) a existência de estudos duplicados, e/ou (iii) estudos detalhados em outras publicações.

\subsection{Resultados e Análise}

Nesse Mapeamento Sistemático foram realizadas buscas automáticas por meio da execução da string de busca nas três fontes de dados. Após isso, foi realizada a triagem dos documentos recuperados.

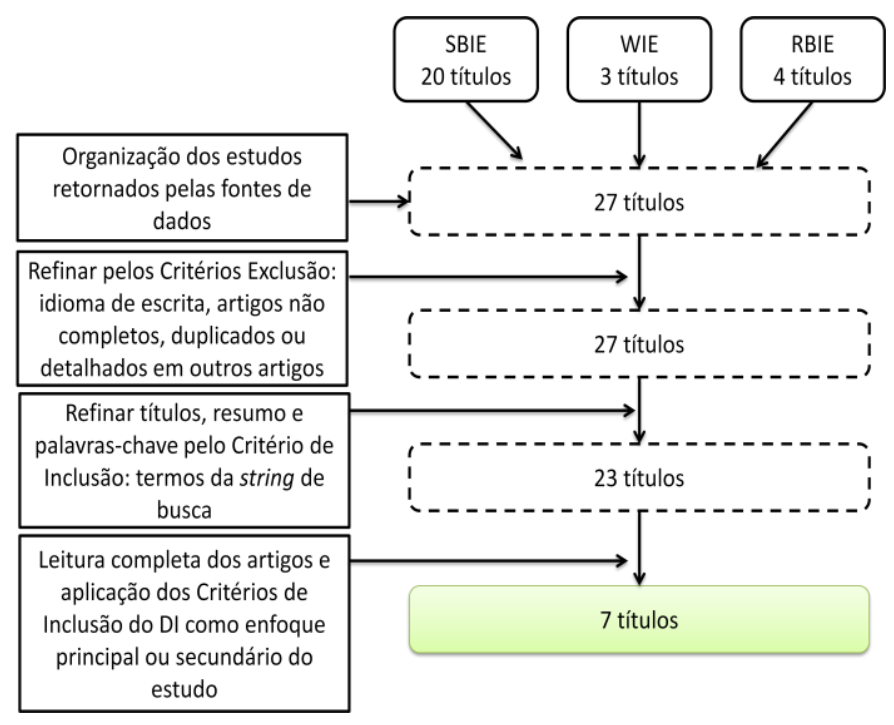

Figura 1. Processo busca e triagem dos documentos

\footnotetext{
${ }^{5}$ Revista Brasileira de Informática na Educação.
} 
A Figura 3 mostra o processo de busca e triagem dos documentos realizados neste trabalho. Durante todo o processo de busca e triagem dos documentos, foram realizados refinamentos sucessivos, com a finalidade de identificar os estudos que mais estivessem de acordo com o objetivo proposto. Ao termino do processo, foram selecionadas 7 publicações, que estão disponíveis em: https://goo.gl/hLBW4r

\subsubsection{Resposta para a questão de pesquisa $n^{0} 1$}

Ao longo do tempo, as pesquisas que abordam o DI, apresentaram variação com relação ao número de publicações. Verifica-se que o DI como planejamento educacional começou a ser utilizado em 2006, com apenas uma publicação, quantidade também identificada nos anos de 2011 e 2014. Porém, nos anos 2010 e 2013 foram realizadas duas publicações/cada, caracterizando-se como os anos com maiores quantidades de publicações (Figura 4). Ressalta-se que as publicações identificadas em 2006 e 2013 foram realizadas no WIE, já as demais ocorreram no SBIE, não havendo, no mesmo ano, publicações em veículos diferentes.

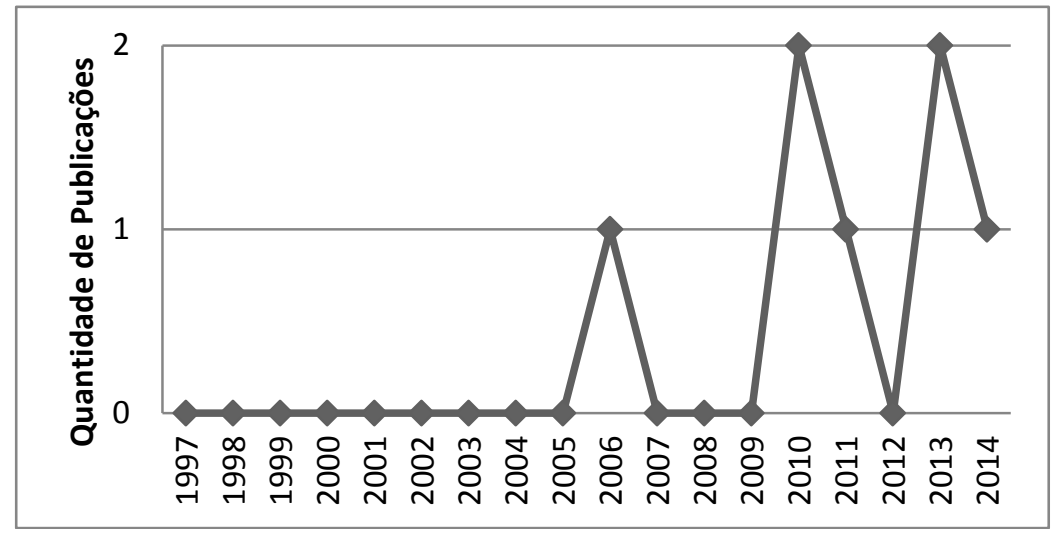

Figura 2. Distribuição quantidade de publicações por ano ${ }^{6}$

\subsubsection{Resposta para a questão de pesquisa $n^{0} 2$}

Inicialmente, para a resolução dessa questão, foram observadas as instituições citadas nos estudos, a fim de classificar os estudos por região e estado, além de quantificar os estudos realizados, conforme detalhamento (Tabela 2).

Tabela 2. Detalhamento das Instituições Identificadas

\begin{tabular}{|c|c|c|c|c|}
\hline Região & Estado & IES & Quantidade & Total \\
\hline \multirow{3}{*}{ Sul } & PR/RS & PUCPR e PUCRS & 1 & \multirow{2}{*}{2} \\
\cline { 2 - 4 } & SC & UDESC e CEAVI & 1 & \\
\hline \multirow{4}{*}{ Nordeste } & \multirow{2}{*}{ CE } & UFC e IFCE & 1 & \multirow{4}{*}{5} \\
\cline { 2 - 4 } & & UFC Virtual & 1 & \\
\cline { 2 - 4 } & BA & UNEB & 1 & \\
\cline { 2 - 4 } & \multirow{2}{*}{ PB } & UFCG & 1 & \\
\cline { 3 - 4 } & & UFCG e FACISA & 1 & \\
\hline
\end{tabular}

${ }^{6} \mathrm{O}$ intervalo de tempo utilizado no gráfico tem como base os anos de publicação da RBIE, por ela apresentar a maior quantidade de anos com estudos publicados. 
CBIE-LACLO 2015

Anais dos Workshops do IV Congresso Brasileiro de Informática na Educação (CBIE 2015)

De acordo com os dados observa-se que, apenas as instituições da região Nordeste e Sul do Brasil contribuíram com publicações que abordaram o uso do DI nos estudos. Quando observado em nível de estados, a Paraíba-PB e o Ceará-CE, ambos da região Nordeste, pontuaram com dois estudos/cada, consequentemente as instituições UFC/UFC Virtual e UFCG destacam-se como as IES que mais participaram dos estudos realizados.

\subsubsection{Resposta para a questão de pesquisa $n^{0} 3$}

Na tabela 3 estão apresentadas as finalidades de uso do DI identificados nos estudos selecionados.

Tabela 3. Objetivo de uso do Design Instrucional nos estudos

\begin{tabular}{|c|c|}
\hline Publicação & Objetivo de uso do DI \\
\hline $\begin{array}{c}\text { A contribuição do Design Instrucional e das Dimensões da } \\
\text { Educação para o desenvolvimento de Objetos de } \\
\text { Aprendizagem }\end{array}$ & $\begin{array}{l}\text { Nortear o planejamento de } \\
\text { Objetos de Aprendizagem }\end{array}$ \\
\hline Aplicação de Learning Analitycs ao Design Instrucional & $\begin{array}{l}\text { Sequenciamento das atividades } \\
\text { de aprendizagem }\end{array}$ \\
\hline $\begin{array}{l}\text { Design Instrucional e seu acompanhamento em tempo de } \\
\text { execução utilizando Rede de Atividades }\end{array}$ & $\begin{array}{l}\text { Modelar e acompanhar atividades } \\
\text { dos estudantes }\end{array}$ \\
\hline $\begin{array}{l}\text { Ferramenta Web para Gerenciamento da Produção de } \\
\text { Objetos de Aprendizagem }\end{array}$ & $\begin{array}{l}\text { Desenvolver de um ambiente de } \\
\text { gerenciamento das atividades de } \\
\text { construção de OA }\end{array}$ \\
\hline $\begin{array}{l}\text { Reestruturação do Curso de Formação em Conselhos } \\
\text { Escolares no AVA Moodle por meio do Design Instrucional }\end{array}$ & Reestruturar um curso EaD Online \\
\hline $\begin{array}{c}\text { Development of a Framework for Quality Instructional } \\
\text { Design Ontologies }\end{array}$ & $\begin{array}{l}\text { Propor um framework para } \\
\text { avaliar da qualidade de ontologias } \\
\text { do Design Instrucional }\end{array}$ \\
\hline Ensino de Design Instrucional: Relatos de Experiência & $\begin{array}{c}\text { Ensinar a disciplina de Design } \\
\text { Instrucional }\end{array}$ \\
\hline
\end{tabular}

De acordo com esse resultado verificam-se diferentes objetivos de uso do DI nas pesquisas realizadas, variando desde a sua utilização para fundamentar as soluções para problemas identificados, até o próprio uso do DI como metodologia e objeto de ensino.

\section{Considerações Finais}

Com a realização desse estudo objetivou-se contribuir com a comunidade IE de duas formas: uma com a finalidade de verificar a aplicação do uso do Design Instrucional como Arquitetura Pedagógica, e caracterizar o uso do Design Instrucional nas publicações de IE no Brasil.

Verificou-se, após mapeamento, que as fases de análise e design do processo de planejamento do DI compreendem todos os elementos e apresentam funcionalidades semelhantes aos elementos de uma AP. Logo, é possível identificar uma conformidade entre as duas abordagens, possibilitando o uso do DI como AP, e, além disso, o DI faz abrange as etapas de desenvolvimento, implementação e avaliação do planejamento realizado. 


\section{CBIE-LACLO 2015}

Anais dos Workshops do IV Congresso Brasileiro de Informática na Educação (CBIE 2015)

Com relação ao MS realizado, foi possível identificar que, nas principais fontes de busca de publicações na área de IE no Brasil, o Design Instrucional não é um tema comumente abordado pelos pesquisadores, pois, apenas sete publicações foram retornadas após todo o processo de busca e triagem.

Ressalta-se que das 27 publicações inicialmente retornadas pelas fontes de busca, 20 foram excluídas durante o processo de triagem. Dessas, foram rejeitadas $100 \%$ das publicações da RBIE (4 artigos) e 80\% das publicações do SBIE (16 artigos), o WIE não apresentou rejeições de publicações. Com isso, dentre os veículos consultados, o SBIE e o WIE apresentam-se em destaque. Quando se observa o nível de aceitação das publicações, verifica-se que o WIE aparece em destaque com todas as publicações aceitas.

A partir das respostas dos resultados e análises verificou-se que grande parte dos estudos selecionados apresentou a utilização do DI de forma secundária, ou seja, seu uso foi para fundamentar o trabalho realizado ou a possível solução do problema de pesquisa identificado.

Também foi observado que apenas as regiões Sul e Nordeste do Brasil realizam publicações nos veículos consultados. A região Nordeste do Brasil destacou-se devido as contribuições realizadas pela UFCG, Campina Grande - PB e a UFC, Fortaleza - CE, que tiveram duas publicações selecionadas para cada IES.

Por fim, espera-se que o mapeamento entre as abordagens e o levantamento do uso do DI nas publicações do DI, objetivos desse trabalho, tenha contribuído para o conhecimento das abordagens aqui estudadas, bem como, proporcione a realização de outros estudos futuros pela comunidade de Informática na Educação.

\section{Referências}

Behar, P. A. e colaboradores (2009). Modelos pedagógicos para a educação à distância. Porto Alegre: Artmed.

Behar, P. A., Bernardi, M., Silva, K. K. L. (2009). Arquiteturas Pedagógicas para a Educação a Distância: a construção e validação de um objeto de aprendizagem. Disponível em: http://seer.ufrgs.br/index.php/renote/article/view/14088/7984. Último acesso em: 30 de julho de 2015.

Bittencourt, I. I. e Costa, E. (2010). Modelos e Ferramentas para Construção de Sistemas Educacionais Adaptativos e Semânticos. XXI Simpósio Brasileiro de Informática na Educação. João Pessoa - PB. 2010.

Carvalho, M. J. S., Nevado, R. A., Menezes, C. S. (2005) Arquiteturas Pedagógicas para Educação a Distância: Concepções e Suporte Telemático. XVI Simpósio Brasileiro de Informática na Educação. Juiz de Fora - MG. 2005

Dallacosta, A.; Cazetta, G.; Souza, S. G de. (2010) Novas tecnologias aplicadas na elaboração de material instrucional online. $3^{\circ}$ Simpósio Hipertexto e Tecnologias na Educação (redes sociais e aprendizagem). Recife - PE.2010.

Fetaji, B; Fetaji, M. (2010) E-Learning Indicators: A Multidimensional Model for Planning Developing and Evaluating E-Learning Software Solutions. In: E-Learning, experiences and future. Edited by Safeeullah Soomro and published by In-Teh. Pag 1- 34.2010 


\section{CBIE-LACLO 2015}

Anais dos Workshops do IV Congresso Brasileiro de Informática na Educação (CBIE 2015)

Filatro, A. Design Instrucional Contextualizado - educação e tecnologia. $3^{\mathrm{a}}$ edição. Editora Senac - São Paulo. 215 páginas. 2010.

Filatro, A. Design Instrucional na Prática. Person Education do Brasil. ISBN: 978-857605-188-6. 173 páginas. 2008.

Hage, H.; Aimeur, E. (2010) E-learning for the new generations, a Web 2.0 approach. In: E-learning. Edited by Marina Buzzi. In-Tech, ISBN:978-953-7619-95-4. 2010.

Kitchenham, B.; Charters, S. "Guidelines for performing systematic literature reviews in software engineering". Keele University and Durham University Joint Report, Tech. Rep. EBSE 2007-001, 2007.

Nevado, R. A., Dalpiaz, M. M., Menezes, C. S. (2009) Arquitetura Pedagógica para Construção Colaborativa de Conceituações. In Anais do $15^{\circ}$ Workshop de Informática na Escola (WIE), Bento Gonçalves, Rio Grande do Sul.

Petersen, K., Feldt, R., Mujtaba, S. and Mattsson, M. (2008) Systematic mapping studies in software engineering. In Proceedings of the international conference on Evaluation and Assessment in Software Engineering, p. 68-77.

\section{Artigos Selecionados no Mapeamento}

Da Silva, C. L. O., De Lima, L., Loureiro, R. C. e Jucá, A. O (2013) Ensino de Design Instrucional: Relatos de Experiência. In Anais do $19^{\circ}$ Workshop de Informática na Escola (WIE), Campinas, São Paulo.

Dos Santos, A. G., Nunes, I. D. e Schiel, U. (2014) Aplicação de Learning Analitycs ao Design Instrucional. In Anais do $25^{\circ}$ Simpósio Brasileiro de Informática na Educação (SBIE), Dourados, Mato Grosso do Sul.

Leite, E. A. M., Sales, G. L., Lima, H. V. e Aguiar, E. P. S. (2013) Reestruturação do Curso de Formação em Conselhos Escolares no AVA Moodle por meio do Design Instrucional. In Anais do $19^{\circ}$ Workshop de Informática na Escola (WIE), Campinas, São Paulo.

Li, X. e Amorim, Ricardo. (2010) Development of a Framework for Quality Instructional Design Ontologies. In Anais do $21^{\circ}$ Simpósio Brasileiro de Informática na Educação (SBIE), João Pessoa, Paraíba.

Nunes, I. D. e Schiel, U. (2011) Design Instrucional e seu acompanhamento em tempo de execução utilizando Rede de Atividades. In Anais do $22^{\circ}$ Simpósio Brasileiro de Informática na Educação (SBIE), Aracajú, Sergipe.

Ramos, A. F. e Dos Santos, P. K. (2006) A contribuição do Design Instrucional e das Dimensões da Educação para o desenvolvimento de Objetos de Aprendizagem. In Anais do $12^{\circ}$ Workshop de Informática na Escola (WIE), Campo Grande, Mato Grosso do Sul.

Vahldick, A. e Knaul, J. C. (2010) Ferramenta Web para Gerenciamento da Produção de Objetos de Aprendizagem. In Anais do $21^{\circ}$ Simpósio Brasileiro de Informática na Educação (SBIE), João Pessoa, Paraíba. 